
\title{
25 Research Square \\ Program Evaluation of a Student-led Peer Support Service at a Canadian University
}

Rahul Suresh ( $\nabla$ rahul.suresh2@mail.mcgill.ca)

Montreal Neurological Institute and Hospital, McGill University https://orcid.org/0000-0002-0805-261X

\section{Zoe Karkossa}

McGill University

Jérémie Richard

McGill University

Maharshee Karia

McGill University

\section{Research}

Keywords: Peer support, university, college, mental health, stress, academic institutions, support

Posted Date: October 30th, 2020

DOl: https://doi.org/10.21203/rs.3.rs-98100/v1

License: (9) This work is licensed under a Creative Commons Attribution 4.0 International License. Read Full License 


\section{Abstract}

Background:University/college campuses are a rigorous academic environment that also contain numerous financial, social and emotional stressors that often result in the deterioration of students' mental health. The Peer Support Centre (PSC) is a pilot project that was established to provide peer support to students in these stressful conditions. We wanted to investigate whether peer support is a viable form of support that would benefit university students. The objective of this study is to determine whether the organization was indeed providing a beneficial service to students and if it was fulfilling the needs of the students that visited the service.

Methods: After a support session, students (also referred to as supportees) were asked to complete an anonymous questionnaire regarding their self-reported mental wellbeing, their experience with previous professional mental health services, and their experience at the PSC. There weren't any selection criteria for either the supportees or volunteers as the completion of the questionnaire was completely voluntary. Additionally, volunteers (also referred to as supporters) were asked to complete a similar questionnaire after conducting a support session. With the data collected from 1043 supportees and 797 volunteers from September 2016 - March 2020, a program evaluation was conducted for quality improvement purposes. The responses to the questionnaires were analysed by calculating the means, modes, standard deviations and performing Two-Sample t-tests.

Results: The PSC is used by a wide variety of students of different sexes, genders, ethnicities. Students reported having a low ORS score, moderate anxiety as per the GAD-7 and moderate depression according to the PHQ-9. They find it easy to use and rely on it as an alternative form of support when they approach barriers that prevent them from accessing professional services. Lastly, the supporters feel very validated in their role and overall quite prepared and helpful when helping their fellow peers.

Conclusions: The established of a service that delivers peer support would be beneficial to the students on a university or college campus as it can serve as a complement to professional services.

\section{Background}

University life can be the source of a host of academic, social, financial and cultural challenges for many students. [1-3] These stressors can lead to worsened academic difficulties and/or mental health issues (e.g. anxiety, depression, poor sleep, eating disorders, substance misuse and abuse and/or suicide), ultimately leading to an overall decrease in one's quality of life. [4-10] Interestingly, 37-84\% of university students who screened positive for depression or anxiety at a university in the US did not consult professional mental health services to address their mental health struggles. [11-13] Moreover, individuals from ages 15 to 24 are the least likely age group to seek aid for their mental health in the form of professional services, despite being the most affected by mental illness in Canada. [14]

The reluctance to seek these professional services can be attributed to the stigma surrounding mental health issues, low perceived need for help, lack of time, lengthy wait times or waitlists, privacy concerns, 
or the hierarchical and illness-based approach conducted by clinicians. [6, 12-16] These barriers can lead university students to rely on informal forms of support such as friends and family to help them cope with mental health distress. [17] Peer-support services can also serve as an alternative source of informal support for university students due to it being free of cost and conveniently situated on-campus. $[17,18]$

Peer support is defined as the social and emotional support offered by an individual in equal standing, founded on respect, shared responsibility and mutual agreement of what is helpful. $[19,20]$ The authenticity found in peer-supporter relationships can lead to greater feelings of empathy and connectedness as compared to a patient-therapist relationship. [21,22] Currently available literature shows how participation in peer support workshops and courses lead to improvements in self-esteem, self-acceptance and overall improvements in mental wellbeing amongst university students. [23, 24] However, the literature on the benefits of a service that delivers peer support is limited due to it being a relatively new phenomenon on university campuses. We sought to investigate the effectiveness of a peer support service developed at a Canadian university and determine whether it is meeting the needs of the students that use it.

\section{Methods}

\section{Peer Support Service}

The Peer Support Centre (PSC) is an on-campus, student-led service established at McGill University in downtown Montreal, Quebec. The PSC works closely with the university's mental health services and professionals to provide free, one-on-one, non-judgemental and non-directional active-listening support to McGill University's student body. PSC consists of over 100 trained peer support volunteers (i.e. supporters) that undergo rigorous training and evaluations to be able to support students during the academic year from September to April. The training program is developed by students based on the community's needs, and focuses on active listening, open communication, empathy, and crisis management (during individuals' disclosures of imminent harm to themselves, others or ongoing child abuse). Confidentiality is an essential mandate of the PSC, with the supporters and supportees being required to sign confidentiality agreements prior to initiating a support session. A support session is a safe space in which the supportee can talk about anything that is on their mind to a supporter who will actively listening in an empathetic, non-judgemental and non-directional manner.

\section{Participants and Procedures}

Program evaluations are within the mandate of PSC, with no addition of questions or interventions outside the scope of the organization, thus ethical approval from the university's Research Ethics Board (REB) was not required (TCPS, Article 2.3 and 2.5). Furthermore, the data collected is completely anonymous and the process of data linkage does not generate any identifiable information, thus any secondary use of data that occurs in this study is permitted without approval from the REB (TCPS, Article 2.4). Participants were recruited through the PSC from September 2016 to March 2020 and included all who accessed the service without any exclusion criteria. After a support session, supportees were invited 
to fill out an anonymous questionnaire via hard copy or a laptop, with their participation being completely voluntary and confidential. Those who consented to fill out the questionnaire were provided a private space and were permitted to not answer any question on the survey that they felt uncomfortable answering.

\section{Measure of Mental Health Status}

To assess supportees' depressive symptoms over the prior two weeks, nine questions from the Patient Health Questionnaire-9 (PHQ-9) were asked. [25] To assess their experience with anxiety symptoms over the last two weeks, seven questions from the Generalized Anxiety Disorder-7 (GAD-7) were asked. [26] The options for the responses to both sets of questions range from 0 ("Not at all") to 3 ("Nearly every day"). The sum of the scores for the PHQ-9 was graded based on which interval it fell into: none/minimal (0-5) to severe (20+). The sum of the scores for the GAD-7 questionnaire was graded according to the following range: minimal $(0-5)$ to severe (15+). Both the PHQ-9 and GAD-7 are considered to be reliable and valid tools for measuring depression and anxiety respectively. [27] A question was also asked to assess how difficult these problems made it for the supportees to get along with other people, to perform academically, and take care of things at home. The responses to these questions range from 0 ("Not difficult at all") to 3 ("Extremely difficult").

Finally, the supportees completed the Outcome Rating Scale (ORS) to assess their personal, interpersonal, social and general wellbeing. [28] The responses to the questions range from 0 ("Low wellbeing") to 10 ("High wellbeing") for each category. The sum of the responses was assessed according to the following intervals: overall low wellbeing (0-24) and average to high wellbeing (25+). The ORS is found to be a valid and reliable tool to assess therapeutic outcomes. [28]

\section{Session Analysis}

In order to assess the quality of the peer support sessions, the Session Rating Scale (SRS) was used. [29] The responses to the four questions can range from 0 ("Low agreement") to 10 ("High agreement"). The sum of the scores were then graded according to the following intervals: potential issues present in the relationship with the supporter (0-35), and good relationship between the supporter and supportee (36+). The SRS is shown to be adequately reliable and valid as a clinical tool. [29]

\section{Qualitative Assessment of PSC}

To assess how students compared the service they received at PSC compared to other mental health services they visited in the past and their experience using the PSC, they were asked qualitative questions/prompts to which they could give a rating from 1 (Terrible) - 5 (Excellent) or "Strongly Disagree" - "Strongly Agree". To assess volunteers' wellbeing after a support sessions and their feelings of preparedness and helpfulness, they were asked to rank their agreement with shown prompts from 1 (Not at all) to 10 (Yes, very) or 1 (Not at all) to 5 (Yes, a lot). A more extensive methodology for PSC's qualitative assessment can be found in the Supplementary Methods. 


\section{Data Analysis}

The significance of changes between the mean score of responses over time was gauged using the TwoSample t-Test assuming either equal or unequal variances based on the result of a F-test on the sample variances. An alpha value of 0.05 was considered significant. Since all of the sample sizes test were large (at least $N>90$ ), we did not test for normality. Data was analyzed using SPSS Statistics, Version 26 (IBM, Armonk, NY) and Microsoft Excel (Microsoft, Redmond, Washington).

\section{Results}

\section{The PSC serves a wide range of students throughout the school year}

From September 2016 to March 2020, the PSC hosted a total of 1164 support sessions with 950 questionnaires being completed, representing a response rate of $81.6 \%$ (Supplementary Table 1). During those four academic years, the PSC was visited by a diverse range of students coming from various academic and ethnic backgrounds, encompassing a variety of genders and sexual orientations (Table 1). Interestingly, students visited this service to a greater extent during the months of October, November, February and March, which coincide with midterm examination periods (Fig. 1A, Supplementary Table 2). 
Table 1

Table showing the demographic breakdown of all the students that used the service from 2016-2020. N $=1043$.

\begin{tabular}{|c|c|c|c|c|c|}
\hline & $\begin{array}{l}2016- \\
2017 \mathrm{n} \\
(\%)\end{array}$ & $\begin{array}{l}2017- \\
2018 \mathrm{n} \\
(\%)\end{array}$ & $\begin{array}{l}2018- \\
2019 \mathrm{n} \\
(\%)\end{array}$ & $\begin{array}{l}2019- \\
2020 \mathrm{n} \\
(\%)\end{array}$ & $\begin{array}{l}\text { Total } \\
2016- \\
2020 \\
\text { n (\%) }\end{array}$ \\
\hline Gender & $116(35.6)$ & $69(24.0)$ & $27(26.2)$ & $38(43.7)$ & \multirow{2}{*}{$\begin{array}{l}250 \\
(31.1)\end{array}$} \\
\hline Male & $205(62.9)$ & $210(73.2)$ & $75(72.8)$ & $44(50.6)$ & \\
\hline Female & $5(1.5)$ & $4(1.4)$ & $3(2.9)$ & $2(2.3)$ & $\begin{array}{l}534 \\
(66.5)\end{array}$ \\
\hline Non-binary & $0(0.0)$ & $0(0.0)$ & $0(0.0)$ & $2(2.3)$ & $\begin{array}{l}14 \\
(1.7)\end{array}$ \\
\hline & $0(0.0)$ & $4(1.4)$ & $0(0.0)$ & $0(0.0)$ & $2(0.2)$ \\
\hline Other & & & & & $4(0.5)$ \\
\hline Sexual Orientation & $14(4.5)$ & $6(2.1)$ & $5(5.2)$ & $12(13.6)$ & \multirow{2}{*}{$\begin{array}{l}37 \\
(2.9)\end{array}$} \\
\hline Homosexual & $248(79.5)$ & $203(71.5)$ & $65(67.0)$ & $61(69.3)$ & \\
\hline Heterosexual & $27(8.7)$ & $58(20.4)$ & $17(17.5)$ & $6(6.8)$ & $\begin{array}{l}577 \\
(42.1)\end{array}$ \\
\hline Bisexual & $14(4.5)$ & $12(4.2)$ & $2(2.1)$ & $1(1.1)$ & \multirow{2}{*}{$\begin{array}{l}108 \\
(10.4)\end{array}$} \\
\hline Pansexual & $3(1.0)$ & $1(0.4)$ & $1(1.0)$ & $0(0.0)$ & \\
\hline Asexual & $4(1.3)$ & $4(1.4)$ & $2(2.1)$ & $0(0.0)$ & $\begin{array}{l}29 \\
(1.9)\end{array}$ \\
\hline Queer & $13(4.2)$ & $12(4.2)$ & $4(4.1)$ & $7(8.0)$ & $5(0.3)$ \\
\hline Questioning/Unsure & $\mathrm{N} / \mathrm{A}$ & $\mathrm{N} / \mathrm{A}$ & $1(1.0)$ & $1(1.1)$ & $\begin{array}{l}10 \\
(0.8)\end{array}$ \\
\hline \multirow{2}{*}{ Prefer not to answer } & & & & & $\begin{array}{l}36 \\
(2.9)\end{array}$ \\
\hline & & & & & $2(0.3)$ \\
\hline
\end{tabular}




\begin{tabular}{|c|c|c|c|c|c|}
\hline & $\begin{array}{l}2016- \\
2017 \mathrm{n} \\
(\%)\end{array}$ & $\begin{array}{l}2017- \\
2018 \mathrm{n} \\
(\%)\end{array}$ & $\begin{array}{l}2018- \\
2019 \mathrm{n} \\
(\%)\end{array}$ & $\begin{array}{l}2019- \\
2020 \mathrm{n} \\
(\%)\end{array}$ & $\begin{array}{l}\text { Total } \\
2016- \\
2020 \\
\text { n (\%) }\end{array}$ \\
\hline Year of Study & $28(8.6)$ & $45(15.6)$ & $9(9.8)$ & $6(6.1)$ & \multirow{2}{*}{$\begin{array}{l}88 \\
(10.9)\end{array}$} \\
\hline U0 & $90(27.7)$ & 59 (20.4) & $10(10.9)$ & $26(26.5)$ & \\
\hline U1 & $58(17.8)$ & $62(21.5)$ & $22(23.9)$ & $26(26.5)$ & $\begin{array}{l}185 \\
(23.0)\end{array}$ \\
\hline U2 & $62(19.1)$ & $65(22.5)$ & $21(22.8)$ & 13 (13.3) & \multirow{2}{*}{$\begin{array}{l}168 \\
(20.9)\end{array}$} \\
\hline U3 & $19(5.8)$ & $27(9.3)$ & $10(10.9)$ & $7(7.1)$ & \\
\hline U4 & $51(15.7)$ & $14(4.8)$ & $12(13.0)$ & 15 (15.3) & $\begin{array}{l}161 \\
(20.0)\end{array}$ \\
\hline Masters & $8(2.5)$ & $10(3.5)$ & $2(2.2)$ & $3(3.1)$ & \multirow{2}{*}{$\begin{array}{l}63 \\
(7.8)\end{array}$} \\
\hline $\mathrm{PhD}$ & $4(1.2)$ & $6(2.1)$ & $3(3.3)$ & $0(0.0)$ & \\
\hline Exchange & $5(1.5)$ & $1(0.3)$ & $3(3.3)$ & $2(2.0)$ & $\begin{array}{l}92 \\
(11.4)\end{array}$ \\
\hline \multirow[t]{3}{*}{ Other } & & & & & $\begin{array}{l}23 \\
(2.9)\end{array}$ \\
\hline & & & & & $\begin{array}{l}13 \\
(1.6)\end{array}$ \\
\hline & & & & & $\begin{array}{l}11 \\
(1.4)\end{array}$ \\
\hline
\end{tabular}




\begin{tabular}{|c|c|c|c|c|c|}
\hline & $\begin{array}{l}2016- \\
2017 \mathrm{n} \\
(\%)\end{array}$ & $\begin{array}{l}2017- \\
2018 \mathrm{n} \\
(\%)\end{array}$ & $\begin{array}{l}2018- \\
2019 \mathrm{n} \\
(\%)\end{array}$ & $\begin{array}{l}2019- \\
2020 \mathrm{n} \\
(\%)\end{array}$ & $\begin{array}{l}\text { Total } \\
2016- \\
2020 \\
\text { n (\%) }\end{array}$ \\
\hline Faculty & $27(8.4)$ & $7(2.4)$ & $2(2.0)$ & $0(0.0)$ & \multirow{2}{*}{$\begin{array}{l}36 \\
(4.5)\end{array}$} \\
\hline Agriculture and Environment & $118(36.8)$ & $128(44.4)$ & $43(43.9)$ & $48(49.0)$ & \\
\hline Arts & $9(2.8)$ & $15(5.2)$ & $0(0.0)$ & $1(1.0)$ & $\begin{array}{l}337 \\
(41.9)\end{array}$ \\
\hline Arts and Science & $0(0.0)$ & $1(0.3)$ & $0(0.0)$ & $0(0.0)$ & \multirow{2}{*}{$\begin{array}{l}25 \\
(3.1)\end{array}$} \\
\hline Dentistry & $11(3.4)$ & $8(2.8)$ & $8(8.2)$ & $5(5.1)$ & \\
\hline Education & $31(9.7)$ & $46(16.0)$ & $8(8.2)$ & $8(8.2)$ & $1(0.1)$ \\
\hline Engineering & $1(0.3)$ & $0(0.0)$ & $4(4.1)$ & $1(1.0)$ & $\begin{array}{l}32 \\
(4.0)\end{array}$ \\
\hline Law & $28(8.7)$ & $20(6.9)$ & $4(4.1)$ & $7(7.1)$ & \multirow{2}{*}{$\begin{array}{l}93 \\
(11.6)\end{array}$} \\
\hline Management & $13(4.0)$ & $7(2.4)$ & $4(4.1)$ & $11(11.2)$ & \\
\hline Medicine & $13(4.0)$ & $6(2.1)$ & $2(2.0)$ & $0(0.0)$ & \multirow{2}{*}{$\begin{array}{l}0(0.1) \\
59 \\
(7.3)\end{array}$} \\
\hline Music & $71(22.1)$ & $56(19.4)$ & $23(23.5)$ & $16(16.3)$ & \\
\hline Science & $0(0.0)$ & $0(0.0)$ & $0(0.0)$ & $1(1.0)$ & $\begin{array}{l}35 \\
(4.3)\end{array}$ \\
\hline \multirow{3}{*}{ Continuing Studies } & & & & & $\begin{array}{l}21 \\
(2.6)\end{array}$ \\
\hline & & & & & $\begin{array}{l}166 \\
(20.6)\end{array}$ \\
\hline & & & & & $1(0.1)$ \\
\hline
\end{tabular}




\begin{tabular}{|c|c|c|c|c|c|}
\hline & $\begin{array}{l}2016- \\
2017 n \\
(\%)\end{array}$ & $\begin{array}{l}2017- \\
2018 n \\
(\%)\end{array}$ & $\begin{array}{l}2018- \\
2019 n \\
(\%)\end{array}$ & $\begin{array}{l}2019- \\
2020 n \\
(\%)\end{array}$ & $\begin{array}{l}\text { Total } \\
2016- \\
2020 \\
\text { n (\%) }\end{array}$ \\
\hline \multirow{3}{*}{$\begin{array}{l}\text { Race/ethnicity } \\
\text { White/Caucasian/European } \\
\text { descent }\end{array}$} & 119 (16.0) & $100(13.4)$ & $39(5.2)$ & $27(3.6)$ & \multirow{2}{*}{$\begin{array}{l}285 \\
(38.2)\end{array}$} \\
\hline & $64(8.6)$ & $65(8.7)$ & $30(4.0)$ & $28(3.8)$ & \\
\hline & $42(5.6)$ & $41(5.5)$ & $9(1.2)$ & $11(1.5)$ & $\begin{array}{l}187 \\
(25.1)\end{array}$ \\
\hline \multirow{3}{*}{$\begin{array}{l}\text { South Asian } \\
\text { West Asian/Middle Eastern }\end{array}$} & $22(2.9)$ & $18(2.4)$ & $1(0.1)$ & $1(0.1)$ & \multirow{2}{*}{$\begin{array}{l}103 \\
(13.8)\end{array}$} \\
\hline & $7(0.9)$ & $16(2.1)$ & $1(0.1)$ & $1(0.1)$ & \\
\hline & $12(1.6)$ & $9(1.2)$ & $5(0.7)$ & $13(1.7)$ & $\begin{array}{l}42 \\
(5.6)\end{array}$ \\
\hline \multirow{2}{*}{ Central/South American } & $11(1.5)$ & $9(1.2)$ & $1(0.1)$ & $3(0.4)$ & \multirow{2}{*}{$\begin{array}{l}25 \\
(3.4)\end{array}$} \\
\hline & $8(1.1)$ & $5(0.7)$ & $3(0.4)$ & $2(0.3)$ & \\
\hline \multirow{3}{*}{$\begin{array}{l}\text { African/Black } \\
\text { South-East Asian } \\
\text { Other/Prefer not to say } \\
\text { Indigenous }\end{array}$} & $3(0.4)$ & $2(0.3)$ & $1(0.1)$ & $0(0.0)$ & $\begin{array}{l}39 \\
(5.2)\end{array}$ \\
\hline & $2(0.3)$ & $2(0.3)$ & $0(0.0)$ & $0(0.0)$ & $\begin{array}{l}28 \\
(3.2)\end{array}$ \\
\hline & \multirow[t]{4}{*}{$2(0.3)$} & \multirow[t]{4}{*}{$1(0.1)$} & \multirow[t]{4}{*}{$8(1.1)$} & $2(0.3)$ & $\begin{array}{l}18 \\
(2.4)\end{array}$ \\
\hline \multirow[t]{3}{*}{ Mixed } & & & & & $6(0.8)$ \\
\hline & & & & & $4(0.5)$ \\
\hline & & & & & $\begin{array}{l}13 \\
(1.7)\end{array}$ \\
\hline \multirow{2}{*}{$\begin{array}{l}\text { In-province, Out-of-province, } \\
\text { International Student Status }\end{array}$} & 90 (19.6) & $71(17.4)$ & 27 (19.9) & $30(20.0)$ & \multirow{2}{*}{$\begin{array}{l}218 \\
(27.3)\end{array}$} \\
\hline & $96(20.9)$ & $88(21.6)$ & 25 (18.4) & $16(10.7)$ & \\
\hline \multirow{2}{*}{$\begin{array}{l}\text { In-province (Quebec) } \\
\text { Out-of-province (Canada) }\end{array}$} & $137(29.8)$ & $124(30.5)$ & $42(30.9)$ & $52(34.7)$ & $\begin{array}{l}225 \\
(28.3)\end{array}$ \\
\hline & $47(10.2)$ & $44(10.8)$ & $12(8.8)$ & $11(7.3)$ & \multirow{2}{*}{$\begin{array}{l}355 \\
(44.5)\end{array}$} \\
\hline \multirow{4}{*}{$\begin{array}{l}\text { International (All) } \\
\text { International (US) } \\
\text { International (France) } \\
\text { International (Other) }\end{array}$} & $10(2.2)$ & $12(2.9)$ & $3(2.2)$ & $4(2.7)$ & \\
\hline & $80(17.4)$ & $68(16.7)$ & 27 (19.9) & $37(24.7)$ & $\begin{array}{l}114 \\
(14.3)\end{array}$ \\
\hline & & & & & $\begin{array}{l}29 \\
(3.6)\end{array}$ \\
\hline & & & & & $\begin{array}{l}212 \\
(26.6)\end{array}$ \\
\hline
\end{tabular}


Notably, as reported by supportees, they mainly came in for a support session simply to talk to another person, due to academic stress, general stress, feeling anxious, and/or because they were feeling down (Fig. 1B, Supplementary Table 3). Interestingly, these session topics were also amongst the top five reported reasons for coming in for a support session during each month of the school year (Supplementary Table 4).

Supportees' mental health measures prior to coming to PSC

From September 2018 - March 2020, supportees were asked how they were doing in four different aspects of their lives on the Outcome Rating Scale (ORS). Based on the responses, 234 (77.2\%) supportees had an overall wellbeing score under 25, which indicates that they are experiencing high distress (Supplementary Table 5). Interestingly, all of the sections of the ORS during 2019-2020 were lower on average than during 2018-2019 ( $<<0.05$, Table 2). 
Table 2

Table showing the supportees' Outcome Rating Scale (ORS), Generalized Anxiety Disorder-7 (GAD-7), Patient Health Questionnaire-9 (PHQ-9), and Session Rating Scale (SRS) measures during each year from 2018-2020. $N=321$. * $P<0.05$.

\begin{tabular}{|c|c|c|c|c|c|c|c|}
\hline \multirow[t]{2}{*}{ ORS } & \multirow[t]{2}{*}{ Area of Wellbeing } & \multicolumn{3}{|c|}{$2018-2019$} & \multicolumn{3}{|c|}{$2019-2020$} \\
\hline & & $\begin{array}{l}\text { Mean } \\
\text { (SD) }\end{array}$ & $\begin{array}{l}\text { Mode } \\
\text { (n) }\end{array}$ & Range & $\begin{array}{l}\text { Mean } \\
\text { (SD) }\end{array}$ & $\begin{array}{l}\text { Mode } \\
\text { (n) }\end{array}$ & Range \\
\hline & Individually * & $\begin{array}{l}5.21 \\
(2.00)\end{array}$ & $\begin{array}{l}5 \\
(42)\end{array}$ & $1-10$ & $\begin{array}{l}4.35 \\
(1.80)\end{array}$ & $\begin{array}{l}5 \\
(21)\end{array}$ & $1-8$ \\
\hline & Interpersonally * & $\begin{array}{l}5.44 \\
(2.00)\end{array}$ & $\begin{array}{l}5 \\
(40)\end{array}$ & $1-10$ & $\begin{array}{l}4.67 \\
(1.87)\end{array}$ & $\begin{array}{l}5 \\
(24)\end{array}$ & $1-8$ \\
\hline & Socially * & $\begin{array}{l}5.17 \\
(2.12)\end{array}$ & $\begin{array}{l}6 \\
(42)\end{array}$ & $1-10$ & $\begin{array}{l}4.42 \\
(1.88)\end{array}$ & $\begin{array}{l}5 \\
(21)\end{array}$ & $1-8$ \\
\hline & Overall * & $\begin{array}{l}5.25 \\
(1.80)\end{array}$ & $\begin{array}{l}5 \\
(61)\end{array}$ & $1-10$ & $\begin{array}{l}4.32 \\
(1.75)\end{array}$ & $\begin{array}{l}5 \\
(23)\end{array}$ & $1-8$ \\
\hline & Total ORS Score * & $\begin{array}{l}21.11 \\
(6.81)\end{array}$ & $\begin{array}{l}20 \\
(19)\end{array}$ & $5-40$ & $\begin{array}{l}17.78 \\
(6.54)\end{array}$ & $\begin{array}{l}23 \\
(9)\end{array}$ & $4-32$ \\
\hline \multirow{8}{*}{ GAD- } & Area of Anxiety & 2018- & 19 & & 2019- & 020 & \\
\hline & & $\begin{array}{l}\text { Mean } \\
\text { (SD) }\end{array}$ & $\begin{array}{l}\text { Mode } \\
\text { (n) }\end{array}$ & Range & $\begin{array}{l}\text { Mean } \\
\text { (SD) }\end{array}$ & $\begin{array}{l}\text { Mode } \\
\text { (n) }\end{array}$ & Range \\
\hline & Nervousness & $\begin{array}{l}1.85 \\
(0.92)\end{array}$ & $\begin{array}{l}1 \text { and } \\
2 \\
(61)\end{array}$ & $0-3$ & $\begin{array}{l}1.90 \\
(0.91)\end{array}$ & $\begin{array}{l}1 \\
(28)\end{array}$ & $0-3$ \\
\hline & Inability to stop worrying & $\begin{array}{l}1.81 \\
(0.96)\end{array}$ & $\begin{array}{l}1 \\
(64)\end{array}$ & $0-3$ & $\begin{array}{l}1.86 \\
(0.97)\end{array}$ & $\begin{array}{l}3 \\
(26)\end{array}$ & $0-3$ \\
\hline & Worrying too much & $\begin{array}{l}1.90 \\
(0.89)\end{array}$ & $\begin{array}{l}2 \\
(64)\end{array}$ & $0-3$ & $\begin{array}{l}1.95 \\
(0.89)\end{array}$ & $\begin{array}{l}1 \text { and } \\
3 \\
(28)\end{array}$ & $0-3$ \\
\hline & Trouble relaxing & $\begin{array}{l}1.74 \\
(0.98)\end{array}$ & $\begin{array}{l}1 \\
(65)\end{array}$ & $0-3$ & $\begin{array}{l}1.82 \\
(0.91)\end{array}$ & $\begin{array}{l}1 \\
(32)\end{array}$ & $0-3$ \\
\hline & $\begin{array}{l}\text { Being so restless it is hard to stand } \\
\text { still }\end{array}$ & $\begin{array}{l}0.92 \\
(1.01)\end{array}$ & $\begin{array}{l}0 \\
(83)\end{array}$ & $0-3$ & $\begin{array}{l}1.01 \\
(1.04)\end{array}$ & $\begin{array}{l}0 \\
(32)\end{array}$ & $0-3$ \\
\hline & Being easily annoyed or irritable & $\begin{array}{l}1.14 \\
(0.97)\end{array}$ & $\begin{array}{l}1 \\
(73)\end{array}$ & $0-3$ & $\begin{array}{l}1.22 \\
(1.04)\end{array}$ & $\begin{array}{l}1 \\
(31)\end{array}$ & $0-3$ \\
\hline
\end{tabular}




\begin{tabular}{|c|c|c|c|c|c|c|}
\hline $\begin{array}{l}\text { Feeling afraid something awful } \\
\text { might happen }\end{array}$ & $\begin{array}{l}1.38 \\
(1.08)\end{array}$ & $\begin{array}{l}1 \\
(57)\end{array}$ & $0-3$ & $\begin{array}{l}1.43 \\
(1.11)\end{array}$ & $\begin{array}{l}1 \\
(31)\end{array}$ & $0-3$ \\
\hline Total anxiety score & $\begin{array}{l}10.71 \\
(5.34)\end{array}$ & $\begin{array}{l}7 \\
(18)\end{array}$ & $0-21$ & $\begin{array}{l}11.21 \\
(5.40)\end{array}$ & $\begin{array}{l}7 \\
(11)\end{array}$ & $0-21$ \\
\hline Impairment severity & $\begin{array}{l}1.63 \\
(0.82)\end{array}$ & $\begin{array}{l}1 \\
(84)\end{array}$ & $0-3$ & $\begin{array}{l}1.72 \\
(0.87)\end{array}$ & $\begin{array}{l}2 \\
(36)\end{array}$ & $0-3$ \\
\hline
\end{tabular}

\section{PHQ- Area of Depression}

2018-2019

2019-2020

$\begin{array}{llllll}\text { Mean } & \text { Mode Range } & \begin{array}{l}\text { Mean } \\ (\mathrm{SD})\end{array} & (\mathrm{n})\end{array} \quad \begin{aligned} & \text { Mode } \\ & (\mathrm{n})\end{aligned}$

Little interest or pleasure in doing

things

1.29
$(0.94)$

$0-3$

$1.27 \quad 1$

$0-3$

(79)

(0.92)

Feeling down, depressed, or hopeless

$\begin{array}{ll}1.50 & 1 \\ (0.95) & \end{array}$

(77)

$0-3$

1.64
$(0.93)$

$0-3$

Trouble falling/staying asleep, sleeping too much

1.49

(1.03)

1

(64)

$0-3$

1.67

(0.99)

$1 \quad 0-3$

(38)

Feeling tired or having little energy

1.63
$(0.93)$

1

8)

Poor appetite or overeating

$\begin{array}{ll}1.15 & 1 \\ (1.02) & (65)\end{array}$

$0-3$

1.80

$(0.94)$

1

$0-3$

(32)

$(65)$

$\begin{array}{lll}1.37 & 1 & 0-3 \\ (1.07) & (34) & \end{array}$

Feeling bad about yourself, or that you're a failure, or have let yourself or your family down

$\begin{array}{llllll}1.16 & 1 & 0-3 & 1.74 & 1 & 0-3 \\ (1.03) & & & (0.96) & (37) & \\ & (67) & & & (37)\end{array}$

Trouble concentrating on things such as reading

$\begin{array}{ll}1.47 & 1 \\ (1.02) & \end{array}$

(64)

$0-3$

1.46
$(0.96)$

$0-3$

(37)

\begin{tabular}{|c|c|c|c|c|c|c|}
\hline $\begin{array}{l}\text { Being so fidgety or restless that you } \\
\text { have been moving around more } \\
\text { than usual }\end{array}$ & $\begin{array}{l}1.04 \\
(1.02)\end{array}$ & $\begin{array}{l}0 \\
(79)\end{array}$ & $0-3$ & $\begin{array}{l}0.85 \\
(1.05)\end{array}$ & $\begin{array}{l}0 \\
(43)\end{array}$ & $0-3$ \\
\hline $\begin{array}{l}\text { Thoughts that you would be better } \\
\text { off dead or of hurting yourself in } \\
\text { some way * }\end{array}$ & $\begin{array}{l}0.41 \\
(1.06)\end{array}$ & $\begin{array}{l}0 \\
(132)\end{array}$ & $0-3$ & $\begin{array}{l}0.70 \\
(1.00)\end{array}$ & $\begin{array}{l}0 \\
(49)\end{array}$ & $0-3$ \\
\hline Total depression score & $\begin{array}{l}11.51 \\
(6.16)\end{array}$ & $\begin{array}{l}5 \text { and } \\
13 \\
(15)\end{array}$ & $0-27$ & $\begin{array}{l}12.50 \\
(6.92)\end{array}$ & $\begin{array}{l}9 \text { and } \\
10 \\
(9)\end{array}$ & $0-27$ \\
\hline Impairment severity & $\begin{array}{l}1.62 \\
(0.83)\end{array}$ & 1 & $0-3$ & $\begin{array}{l}1.61 \\
(0.87)\end{array}$ & 1 & $0-3$ \\
\hline
\end{tabular}


SRS Aspect of the Session

\begin{tabular}{|c|c|c|c|c|c|c|}
\hline & $\begin{array}{l}\text { Mean } \\
\text { (SD) }\end{array}$ & $\begin{array}{l}\text { Mode } \\
\text { (n) }\end{array}$ & Range & $\begin{array}{l}\text { Mean } \\
\text { (SD) }\end{array}$ & $\begin{array}{l}\text { Mode } \\
\text { (n) }\end{array}$ & Range \\
\hline \multirow{2}{*}{$\begin{array}{l}\text { Relationship - I felt heard, } \\
\text { understood, and respected. }\end{array}$} & 9.07 & 10 & \multirow[t]{2}{*}{$1-10$} & \multirow{2}{*}{$\begin{array}{l}9.20 \\
(1.10)\end{array}$} & 10 & \multirow[t]{2}{*}{$5-10$} \\
\hline & (1.58) & (126) & & & (55) & \\
\hline \multirow{2}{*}{$\begin{array}{l}\text { Topics - We talked about what I } \\
\text { wanted to talk about. }\end{array}$} & 9.21 & 10 & \multirow[t]{2}{*}{$1-10$} & \multirow{2}{*}{$\begin{array}{l}9.46 \\
(1.08)\end{array}$} & 10 & \multirow[t]{2}{*}{$5-10$} \\
\hline & (1.34) & (122) & & & (71) & \\
\hline \multirow{2}{*}{$\begin{array}{l}\text { Approach or Method - The peer- } \\
\text { supporter's approach was a good fit } \\
\text { for me. }\end{array}$} & 8.56 & 10 & \multirow[t]{2}{*}{$2-10$} & \multirow{2}{*}{$\begin{array}{l}8.72 \\
(1.60)\end{array}$} & 10 & \multirow[t]{2}{*}{$4-10$} \\
\hline & (1.78) & (96) & & & (45) & \\
\hline \multirow{2}{*}{$\begin{array}{l}\text { Relationship - I felt heard, } \\
\text { understood, and respected. }\end{array}$} & 9.07 & 10 & \multirow[t]{2}{*}{$1-10$} & \multirow{2}{*}{$\begin{array}{l}9.20 \\
(1.10)\end{array}$} & 10 & \multirow[t]{2}{*}{$5-10$} \\
\hline & $(1.58)$ & (126) & & & (55) & \\
\hline \multirow{2}{*}{$\begin{array}{l}\text { Topics - We talked about what I } \\
\text { wanted to talk about. }\end{array}$} & 9.21 & 10 & \multirow[t]{2}{*}{$1-10$} & \multirow{2}{*}{$\begin{array}{l}9.46 \\
(1.08)\end{array}$} & 10 & \multirow[t]{2}{*}{$5-10$} \\
\hline & (1.34) & (122) & & & (71) & \\
\hline
\end{tabular}

In order to gauge their levels of anxiety, supportees were asked questions regarding their level of anxiety over the last 2 weeks. Based on their responses to the GAD-7, 34 (12.9\%) of supportees reported experiencing minimal anxiety, 81 (30.8\%) reported mild anxiety, 85 (32.3\%) reported moderate anxiety, and $63(24.0 \%)$ reported severe anxiety (Supplementary Table 5). None of the areas of anxiety were significantly different from 2018-2019 to 2019-2020 (Table 2).

Next, we sought to gain insight on their levels of depression and feeling low in the two weeks prior to coming to the PSC based on the PHQ-9. Overall, 162 (59.5\%) reported experiencing moderate levels of depression or worse (Supplementary Table 5) Notably, the proportion of supportees feeling as though they would be better off dead or hurt in some way was significantly higher during 2019-2020 than 2018-2019 ( $<<0.05$, Table 2).

\section{PSC as an alternative source of support for students' mental health needs}

In order to see how students addressed their low levels of mental wellbeing, they were asked questions regarding their experience with other mental health services. It was reported from September 2016 - April 2019 that $398(60.4 \%)$ students coming into the PSC do not consult any other professional service (Fig. 2A, Supplementary Table 6). Furthermore, 69.7\% $(n=182)$ of those students who are using another professional mental service are on a waitlist either at the McGill campus and/or elsewhere off-campus (Fig. 2B, Supplementary Table 7). Additionally, from September 2018 - March 2020, 87.2\% $(n=231)$ of supportees rated their experience at the PSC from Good to Excellent compared to other mental health 
services that they were accessing, and only $2.3 \%(n=6)$ of supportees reported it as being Terrible or Poor. (Fig. 2C, Supplementary Table 8).

\section{Supportees' opinions on their visit to the PSC}

From September 2018 to March 2020, the mean rating of sessions based on the SRS was found to be 35.9 (S.D. = 5.63), with a mode of $40(n=202)$, which indicates high peer support session quality (Table 2 , Supplementary Table 5). Additionally, 269 (88.8\%) supportees felt that their visit to the PSC helped them with their emotional and mental wellbeing, with a mean of 4.2 (S.D. $=0.73)$ and a mode of $4(n=158$, Supplementary Table 10). From 2016-2018, 548 (91.6\%) supportees felt that the peer supporter understood what they were experiencing, $456(76.5 \%)$ felt that their peer supporter helped them realized their own resilience and/or coping skills, $420(70.9 \%)$ felt that they were pointed towards other possible resources or services in a helpful way, and 453 (76.1\%) felt more equipped to face their circumstances after their support session (Supplementary Figs. 1A - D, Supplementary Table 11). From 2018-2020, 249 (84.9\%) found it relatively simple to navigate the PSC service, 174 (65.7\%) didn't feel that there were many barriers associated with accessing the PSC, and 249 (89\%) perceived the PSC as being beneficial to other students on campus (Supplementary Figs. 1E - G, Supplementary Table 12). Overall, from 20162020, 820 (93.5\%) supportees would recommend the PSC as a service to a friend or a classmate (Supplementary Fig. 1H, Supplementary Table 13).

\section{Volunteers' ability to provide support and their post-session well-being}

Data on the supporters' post-session wellbeing was collected from September 2016 - March 2020, with a response rate of $68.5 \%(n=797$, Supplementary Table 13). In terms of preparedness, $698(88.2 \%)$ supporters felt quite prepared for the topics that came up during the session, with only $16(2.0 \%)$ supporters having felt unprepared (Supplementary Table 14). In terms of helpfulness, 540 (73.7\%) supporters felt that they were very helpful, with only 29 (4.0\%) not feeling helpful to the supportee (Supplementary Table 14). There was no statistically significant change in the supporters' feelings of helpfulness from 2018-2019 to 2019-2020 (Supplementary Table 14). According to the responses from September 2018 - March 2020, after a session, supporters felt very validated in their role $(8.22 \pm 1.61$, mean \pm SD on a scale from $1-10$ ) and rarely felt conflicted about blurring the line between being a supporter and being a potential friend $(2.49 \pm 2.00$, Table 3$)$. 
Table 3

Table of volunteers' mental wellbeing scores from 2018-2020. $N=345$. * $P<0.05$.

\begin{tabular}{|c|c|c|c|c|c|c|c|c|c|}
\hline \multirow[t]{2}{*}{$\begin{array}{l}\text { Area of } \\
\text { Supporter's } \\
\text { Wellbeing }\end{array}$} & \multicolumn{3}{|c|}{ 2018-2019 } & \multicolumn{3}{|c|}{$2019-2020$} & \multicolumn{3}{|c|}{$\begin{array}{l}\text { Total } \\
(2018-2020)\end{array}$} \\
\hline & $\begin{array}{l}\text { Mean } \\
\text { (SD) }\end{array}$ & $\begin{array}{l}\text { Mode } \\
\text { (n) }\end{array}$ & Range & $\begin{array}{l}\text { Mean } \\
\text { (SD) }\end{array}$ & $\begin{array}{l}\text { Mode } \\
\text { (n) }\end{array}$ & Range & $\begin{array}{l}\text { Mean } \\
\text { (SD) }\end{array}$ & $\begin{array}{l}\text { Mode } \\
\text { (n) }\end{array}$ & Range \\
\hline $\begin{array}{l}\text { I feel validated } \\
\text { in my role as a } \\
\text { supporter. }\end{array}$ & $\begin{array}{l}8.22 \\
(1.62)\end{array}$ & $\begin{array}{l}10 \\
(50)\end{array}$ & $3-10$ & $\begin{array}{l}8.24 \\
(1.60)\end{array}$ & $\begin{array}{l}9 \\
(41)\end{array}$ & $2-10$ & $\begin{array}{l}8.22 \\
(1.61)\end{array}$ & $\begin{array}{l}10 \\
(85)\end{array}$ & $2-10$ \\
\hline $\begin{array}{l}\text { I felt conflicted } \\
\text { about how } \\
\text { much advice to } \\
\text { give. }\end{array}$ & $\begin{array}{l}3.99 \\
(2.68)\end{array}$ & $\begin{array}{l}1 \\
(29)\end{array}$ & $1-10$ & $\begin{array}{l}3.71 \\
(2.57)\end{array}$ & $\begin{array}{l}2 \\
(32)\end{array}$ & $2-10$ & $\begin{array}{l}3.88 \\
(2.64)\end{array}$ & $\begin{array}{l}1 \\
(75)\end{array}$ & $1-10$ \\
\hline $\begin{array}{l}\text { I felt conflicted } \\
\text { about being a } \\
\text { supporter vs. } \\
\text { being a } \\
\text { potential friend. } \\
\star\end{array}$ & $\begin{array}{l}2.70 \\
(2.14)\end{array}$ & $\begin{array}{l}1 \\
(86)\end{array}$ & $1-10$ & $\begin{array}{l}2.15 \\
(1.69)\end{array}$ & $\begin{array}{l}1 \\
(64)\end{array}$ & $1-9$ & $\begin{array}{l}2.49 \\
(2.00)\end{array}$ & $\begin{array}{l}1 \\
(150)\end{array}$ & $1-10$ \\
\hline $\begin{array}{l}\text { I feel frustrated } \\
\text { or sad that I } \\
\text { may not see the } \\
\text { supportee again } \\
\text { and see how } \\
\text { they will be in } \\
\text { the future. }\end{array}$ & $\begin{array}{l}3.63 \\
(2.49)\end{array}$ & $\begin{array}{l}1 \\
(55)\end{array}$ & $1-10$ & $\begin{array}{l}3.74 \\
(2.61)\end{array}$ & $\begin{array}{l}1 \\
(32)\end{array}$ & $1-10$ & $\begin{array}{l}3.67 \\
(2.53)\end{array}$ & $\begin{array}{l}1 \\
(87)\end{array}$ & $1-10$ \\
\hline $\begin{array}{l}\text { I felt out of my } \\
\text { depth because } \\
\text { of the intensity } \\
\text { of the } \\
\text { supportees } \\
\text { feelings or } \\
\text { needs. }\end{array}$ & $\begin{array}{l}2.71 \\
(2.14)\end{array}$ & $\begin{array}{l}1 \\
(87)\end{array}$ & $1-10$ & $\begin{array}{l}2.47 \\
(1.96)\end{array}$ & $\begin{array}{l}1 \\
(61)\end{array}$ & $1-9$ & $\begin{array}{l}2.62 \\
(2.07)\end{array}$ & $\begin{array}{l}1 \\
(148)\end{array}$ & $1-10$ \\
\hline $\begin{array}{l}\text { I am worried } \\
\text { about the safety } \\
\text { of my } \\
\text { supportee. }\end{array}$ & $\begin{array}{l}1.94 \\
(1.57)\end{array}$ & $\begin{array}{l}1 \\
(125)\end{array}$ & $1-8$ & $\begin{array}{l}2.15 \\
(1.750\end{array}$ & $\begin{array}{l}1 \\
(69)\end{array}$ & $1-10$ & $\begin{array}{l}2.02 \\
(1.64)\end{array}$ & $\begin{array}{l}1 \\
(194)\end{array}$ & $1-10$ \\
\hline
\end{tabular}

\section{Discussion}

Through this program evaluation, it is evident that the PSC is accessed by a diverse range of individuals. Notably, more females used the service than males, which can in part be explained by females making up a larger part of the student body than males at McGill. [30] This could also be due to males' greater perceived self-stigma surrounding help-seeking behaviours, lower mental health literacy and conflicting ideas about masculinity. [31-34] Additionally, the PSC is visited by a larger proportion of non-White and international students compared to White or Canadian residents, which could be due to them having 
smaller prior support networks, experiencing more difficulty adjusting to their new sociocultural environment, and potentially experiencing more discrimination than their Caucasian peers. [35, 36] Interestingly, a much larger proportion of bisexual individuals and a lower proportion of heterosexual individuals visited the PSC relative to the McGill population, [37] which could possibly be because sexual minority groups tend to face more discrimination and victimization than other groups. [38] These findings suggest that PSC should work to increase perception of service accessibility, as well as continue to enforce and advertise their open, welcoming and non-judgemental mandate to ensure that they are reaching out to a greater number of students and demographic groups in need.

The greater frequency of sessions during midterm examination months is consistent with studies showing that decreased classmate and teacher support correlates with increased mental health difficulties in students, and thus this could be a potential reason for students seeking support from a peer-based service. [39] In terms of student wellness, each aspect of the ORS measure declined going from 2018-2019 to 2019-2020, and although there wasn't a drastic change at McGill in terms of how classes were run, studies have shown that depressive symptoms and burnout are increasing amongst college students. [40] This is also consistent with our findings of a larger number of upper-year university students using our service as compared to first-year students. This finding may be preliminary evidence indicating that rates of mental health among university students are indeed decreasing over time. Unfortunately, there was an increase in the proportion of students with suicidal thoughts, and this still remains a critical issue in academic environments which needs to be addressed by academic institutions. [41]

Consistent with previous reports, most students do not consult any other professional services to address their mental health needs. This could be due to misconceptions regarding the cost and effectiveness of mental health care. [42] A large percentage of students reported not being on a waitlist or not seeing a professional in the first place. Supportees rate PSC very highly and easy to access with minimal barriers compared to other mental health services that they have used, which corroborates with previous studies emphasizing the benefits of peer support. [12-15, 43,44] However, that is to say that peer support serves as a complement to the available professional mental health services rather than replacing them entirely.

Lastly, peer support also benefits supporters through improving their self-esteem and giving them feelings of empowerment. [45] Overall, supporters felt as though they were fulfilling their role as a peer supporter quite well and felt that they were able to help the supportee. Interestingly, supporters felt more prepared in 2018-2019 compared to 2019-2020 ( $P<0.05$, Supplementary Table 15), which may be due to improvements over the years with regards to the PSC's training programs, in addition to improved supplemental trainings and more frequent practice sessions throughout the year.

\section{Limitations}

Supportees consented to fill out the form under the condition that they were freely allowed to not answer questions that they weren't comfortable answering (e.g. ethnicity, their use of other mental health 
services, etc.), so some questions have a smaller number of responses compared to others. Furthermore, since data is anonymous, the same supportee could have completed the questionnaire multiple times and we are unable to monitor this. Additionally, the responses are indicative the short-term effects of a support session, and further studies are required to determine its long-term effects on the mental health of university students. Next, different qualitative questions were asked to the supportees in 2016-2018 as compared to 2018-2020 since the post-session questions were chosen by the Executive team of that year. However, all the data used in this study was collected over a minimum period of 2 years for a sufficient sample size and response rate. Lastly, It should be noted that from 2019-2020, the dataset only spans till mid-March due to the abrupt campus shutdown as a result of COVID-19.

\section{Conclusion}

Peer support and the establishment of on-campus peer support services can be very beneficial to university and college students in helping them better cope with the numerous stressors in their academic environment. The Peer Support Centre appears to fulfil its mandate of providing empathetic, confidential, non-judgemental and non-directional support to students at McGill University in an accessible manner. Taken together, the establishment of an on-campus peer support service is beneficial and relied upon by students at a university campus.

\section{Declarations}

\section{Ethics approval and consent to participate}

Program evaluations are within the mandate of PSC, with no addition of questions or interventions outside the scope of the organization, thus ethical approval from the university's Research Ethics Board (REB) was not required (TCPS, Article 2.3 and 2.5). Furthermore, the data collected is completely anonymous and the process of data linkage does not generate any identifiable information, thus any secondary use of data that occurs in this study is permitted without approval from the REB (TCPS, Article 2.4). Participants were recruited through the PSC from September 2016 to March 2020 and included all who accessed the service without any exclusion criteria. After a support session, supportees were invited to fill out an anonymous questionnaire via hard copy or a laptop, with their participation being completely voluntary and confidential. Those who consented to fill out the questionnaire were provided a private space and were permitted to not answer any question on the survey that they felt uncomfortable answering.

\section{Consent for publication}

Not applicable.

\section{Availability of data and materials}


All data generated or analysed during this study are included in this published article [and its supplementary information files].

\section{Competing interests}

The authors declare that they have no competing interests.

\section{Funding}

This study was conducted completely on a volunteer-basis without any funding.

\section{Authors' contributions}

RS did most of the writing of the paper, created the tables and figures and analyzed the data. ZK aided in writing the manuscript, collected the data as well as performed the statistical analysis. JR aided in writing the paper and edited the paper. MK aided by editing the manuscript. All authors read and approved the final manuscript.

\section{Acknowledgements}

The authors would like to thank the Student Society of McGill University for allowing the establishment of the PSC and recognizing it as an official student organization of the McGill community.

\section{References}

1. Beiter, R., et al., The prevalence and correlates of depression, anxiety, and stress in a sample of college students. J Affect Disord, 2015. 173: p. 90-6.

2. Pierceall, E. and M. Keim, Stress and Coping Strategies Among Community College Students. Community College Journal of Research and Practice, 2007. 31: p. 703-712.

3. Vaez, M. and L. Laflamme, Experienced stress, psychological symptoms, self-rated health and academic achievement: A longitudinal study of Swedish university students. Social Behavior and Personality: an international journal, 2008. 36: p. 183-196.

4. Tang, F., M. Byrne, and P. Qin, Psychological distress and risk for suicidal behavior among university students in contemporary China. J Affect Disord, 2018. 228: p. 101-108.

5. Krumrei, E., F. Newton, and E. Kim, A Multi-Institution Look at College Students Seeking Counseling: Nature and Severity of Concerns. Journal of College Student Psychotherapy, 2010. 24: p. 261-283.

6. Hunt, J. and D. Eisenberg, Mental health problems and help-seeking behavior among college students. J Adolesc Health, 2010. 46(1): p. 3-10. 
7. Auerbach, R.P., et al., Mental disorders among college students in the World Health Organization World Mental Health Surveys. Psychol Med, 2016. 46(14): p. 2955-2970.

8. Regehr, C., et al., Interventions to reduce the consequences of stress in physicians: a review and meta-analysis. J Nerv Ment Dis, 2014. 202(5): p. 353-9.

9. Lee, D., et al., The effects of college counseling services on academic performance and retention. Journal of College Student Development, 2009. 50(3): p. 305-319.

10. Kadison, R. and T.F. DiGeronimo, College of the overwhelmed: The campus mental health crisis and what to do about it. College of the overwhelmed: The campus mental health crisis and what to do about it. 2004, San Francisco, CA, US: Jossey-Bass. vi, 296-vi, 296.

11. Pelletier, L., et al., Under-diagnosis of mood disorders in Canada. Epidemiol Psychiatr Sci, 2017. 26(4): p. 414-423.

12. Eisenberg, D., et al., Mental health service utilization among college students in the United States. J Nerv Ment Dis, 2011. 199(5): p. 301-8.

13. Eisenberg, D., E. Golberstein, and S.E. Gollust, Help-seeking and access to mental health care in a university student population. Med Care, 2007. 45(7): p. 594-601.

14. Cyr, C., Making the Case for Peer Support: Report to the Peer Support Project Committee of the Mental Health Commission of Canada. 2016, The Mental Health Commission of Canada.

15. Gulliver, A., et al., Privacy Issues in the Development of a Virtual Mental Health Clinic for University Students: A Qualitative Study. JMIR Ment Health, 2015. 2(1): p. e9.

16. McKinney, K., Initial Evaluation of Active Minds: A Student Organization Dedicated to Reducing the Stigma of Mental IIIness. Journal of College Student Psychotherapy, 2009. 23: p. 281-301.

17. Ryan, M.L., I.M. Shochet, and H.M. Stallman, Universal online interventions might engage psychologically distressed university students who are unlikely to seek formal help. Advances in Mental Health, 2010. 9(1): p. 73-83.

18. Hefner, J. and D. Eisenberg, Social support and mental health among college students. Am J Orthopsychiatry, 2009. 79(4): p. 491-9.

19. Solomon, P., Peer support/peer provided services underlying processes, benefits, and critical ingredients. Psychiatr Rehabil J, 2004. 27(4): p. 392-401.

20. Ruddon, R.W., What Makes a Cancer Cell a Cancer Cell? 6th edition ed. Cancer Medicine. 2003, Hamilton (ON): BC Decker.

21. Chinman, M., et al., Toward the implementation of mental health consumer provider services. $J$ Behav Health Serv Res, 2006. 33(2): p. 176-95.

22. Coatsworth-Puspoky, R., C. Forchuk, and C. Ward-Griffin, Peer support relationships: an unexplored interpersonal process in mental health. J Psychiatr Ment Health Nurs, 2006. 13(5): p. 490-7.

23. Byrom, N., An evaluation of a peer support intervention for student mental health. J Ment Health, 2018. 27(3): p. 240-246. 
24. Aladag, M. and E. Tezer, Effects of a Peer Helping Training Program on Helping Skills and SelfGrowth of Peer Helpers. International Journal for the Advancement of Counselling, 2009. 31: p. 255269.

25. Kroenke, K., R.L. Spitzer, and J.B. Williams, The PHQ-9: validity of a brief depression severity measure. J Gen Intern Med, 2001. 16(9): p. 606-13.

26. Lowe, B., et al., Validation and standardization of the Generalized Anxiety Disorder Screener (GAD-7) in the general population. Med Care, 2008. 46(3): p. 266-74.

27. Kroenke, K., et al., The Patient Health Questionnaire Somatic, Anxiety, and Depressive Symptom Scales: a systematic review. Gen Hosp Psychiatry, 2010. 32(4): p. 345-59.

28. Bringhurst, D., et al., The Reliability and Validity of the Outcome Rating Scale: A Replication Study of a Brief Clinical Measure. Journal of Brief Therapy, 2006. 5: p. 14.

29. Duncan, B., et al., The Session Rating Scale: Preliminary Psychometric Properties of a "Working" Alliance Measure. Journal of Brief Therapy, 2003. 3: p. 3-12.

30. University, M. Total (FT and PT) Enrolments by Faculty, by Degree and by Gender. 2018 [cited 2020 September 10]; Available from: https://www.mcgill.ca/es/registration-statistics/fall-2018.

31. Latalova, K., D. Kamaradova, and J. Prasko, Perspectives on perceived stigma and self-stigma in adult male patients with depression. Neuropsychiatr Dis Treat, 2014. 10: p. 1399-405.

32. Matheson, F.I., et al., Physical health and gender as risk factors for usage of services for mental illness. J Epidemiol Community Health, 2014. 68(10): p. 971-8.

33. Rafal, G., A. Gatto, and R. DeBate, Mental health literacy, stigma, and help-seeking behaviors among male college students. Journal of American College Health, 2018. 66(4): p. 284-291.

34. Ogrodniczuk, J., et al., Men's mental health: Spaces and places that work for men. Can Fam Physician, 2016. 62(6): p. 463-4.

35. McGarvey, A., et al., International students' experience of a western medical school: a mixed methods study exploring the early years in the context of cultural and social adjustment compared to students from the host country. BMC Med Educ, 2015. 15: p. 111.

36. Ozer, S., Predictors of international students' psychological and sociocultural adjustment to the context of reception while studying at Aarhus University, Denmark. Scand J Psychol, 2015. 56(6): p. 717-25.

37. University, M. Student Demographic Survey. 2009; Available from: https://www.mcgill.ca/studentlifeandlearning/files/studentlifeandlearning/final_report_1.pdf? source=post_page

38. Meyer, I.H., Prejudice, social stress, and mental health in lesbian, gay, and bisexual populations: conceptual issues and research evidence. Psychol Bull, 2003. 129(5): p. 674-697.

39. Wit, D.J.D., et al., Perceptions of declining classmate and teacher support following the transition to high school: Potential correlates of increasing student mental health difficulties. Psychology in the Schools, 2011. 48(6): p. 556-572. 
40. Grace, M.K., Depressive symptoms, burnout, and declining medical career interest among undergraduate pre-medical students. Int J Med Educ, 2018. 9: p. 302-308.

41. Dyrbye, L.N., et al., Burnout and suicidal ideation among U.S. medical students. Ann Intern Med, 2008. 149(5): p. 334-41.

42. Etheredge, J.A., Misperceptions behind mental health policy. JAMA, 2002. 287(14): p. 1858.

43. Davidson, L., et al., Peer support among adults with serious mental illness: a report from the field. Schizophr Bull, 2006. 32(3): p. 443-50.

44. Giamos, D., et al., Understanding Campus Culture and Student Coping Strategies for Mental Health Issues in Five Canadian Colleges and Universities. Canadian Journal of Higher Education / Revue canadienne d\&\#x27;enseignement supérieur, 2017. 47(3): p. 136-151.

45. Bracke, P., W. Christiaens, and M. Verhaeghe, Self-esteem, self-efficacy, and the balance of peer support among persons with chronic mental health problems. Journal of Applied Social Psychology, 2008. 38(2): p. 436-459.

\section{Figures}


A.

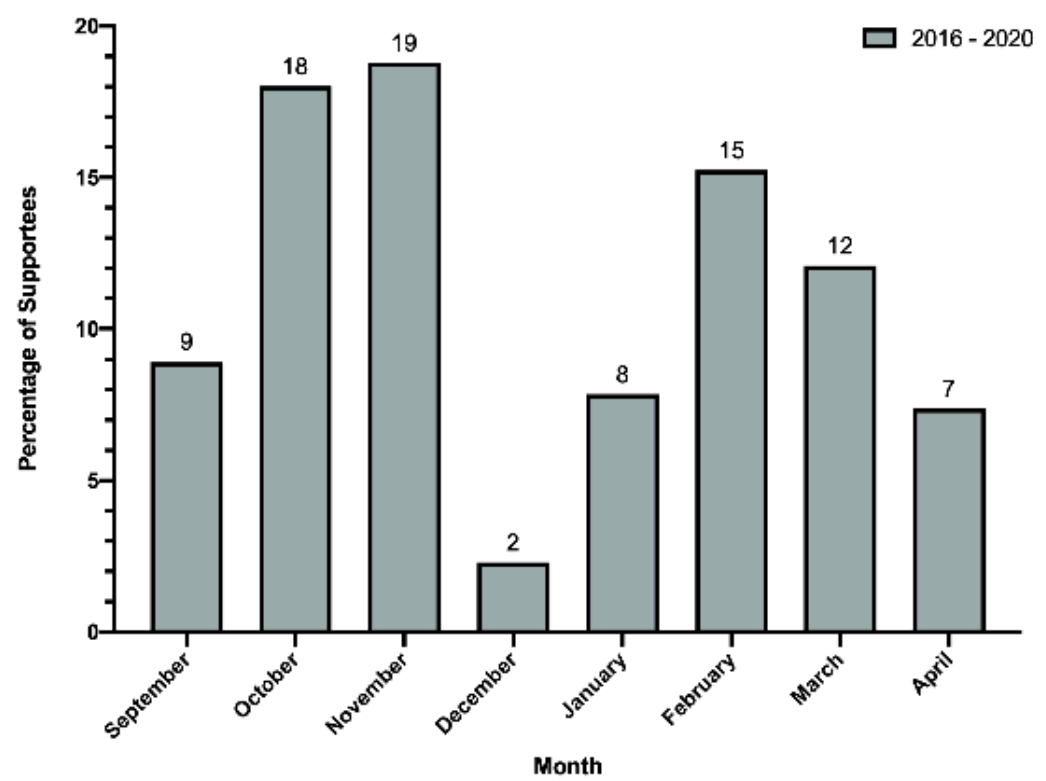

B.

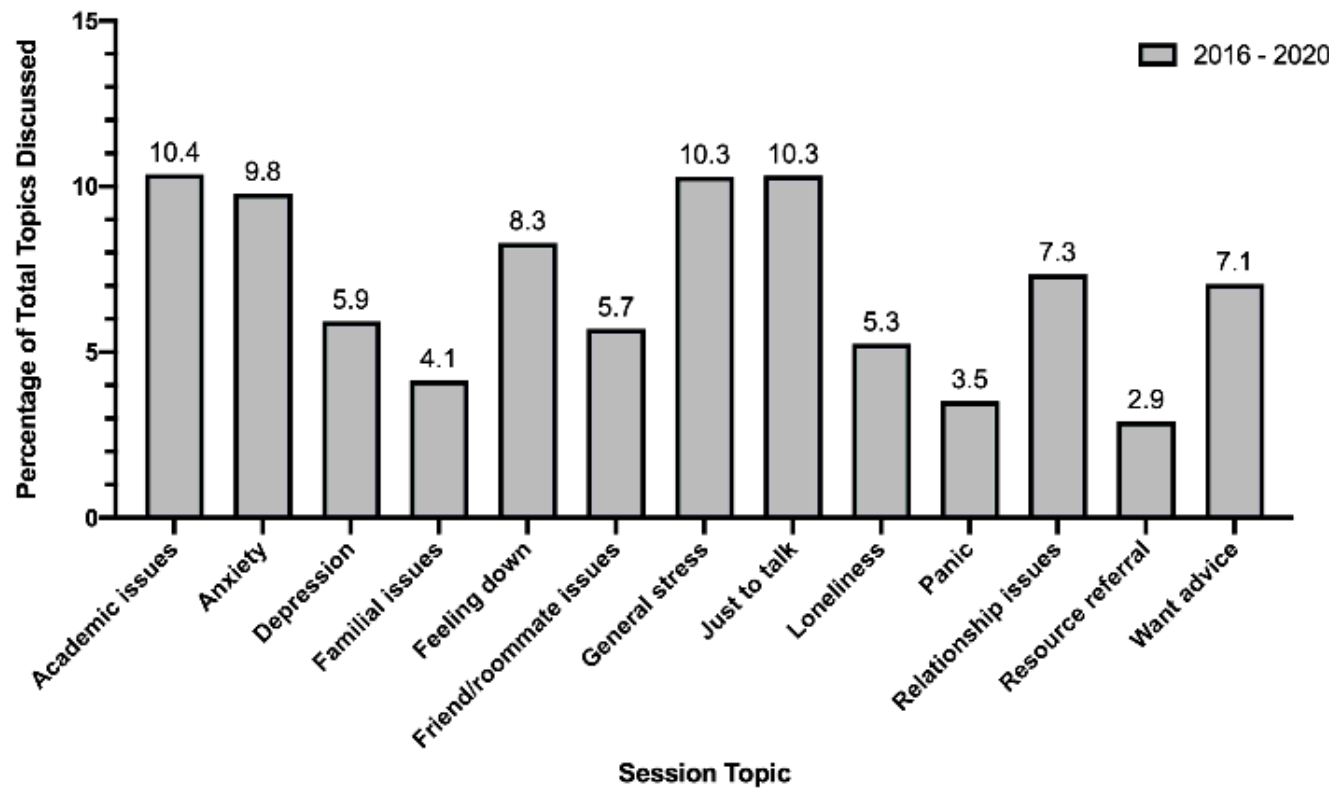

Figure 1

A) Distribution of the percentage of sessions by month of the academic year from $2016-2020$. B) Distribution of topics that came up most (above 2\%) in support sessions from 2016 - 2020 (full list can be found in Supplementary Table 3). 
A.

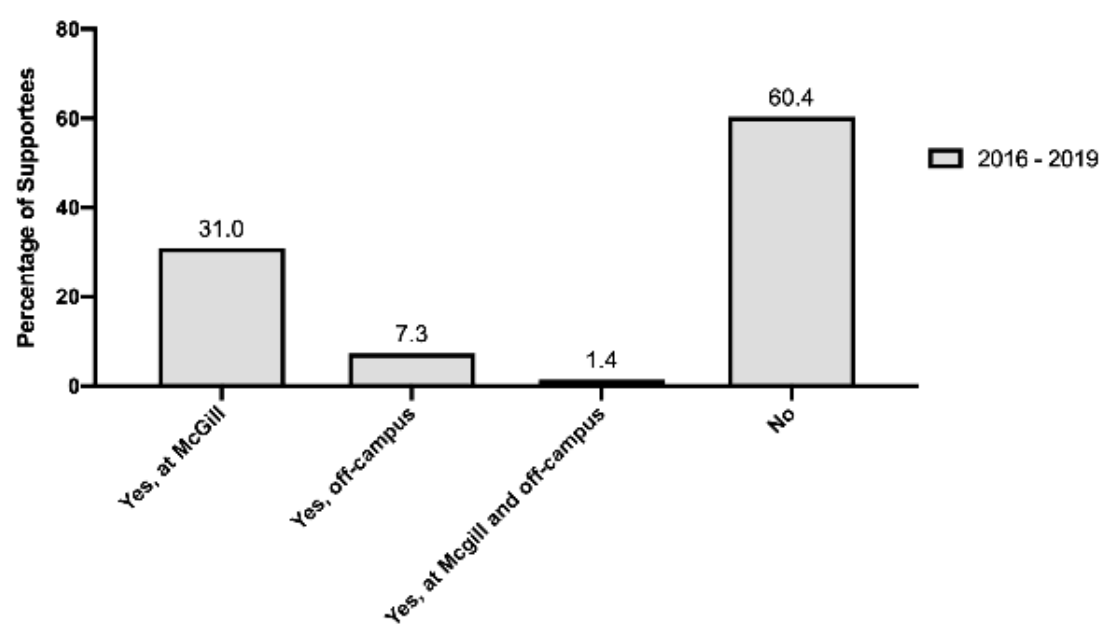

B.

Waitlist Status of Supportees at Professional Mental Health Services

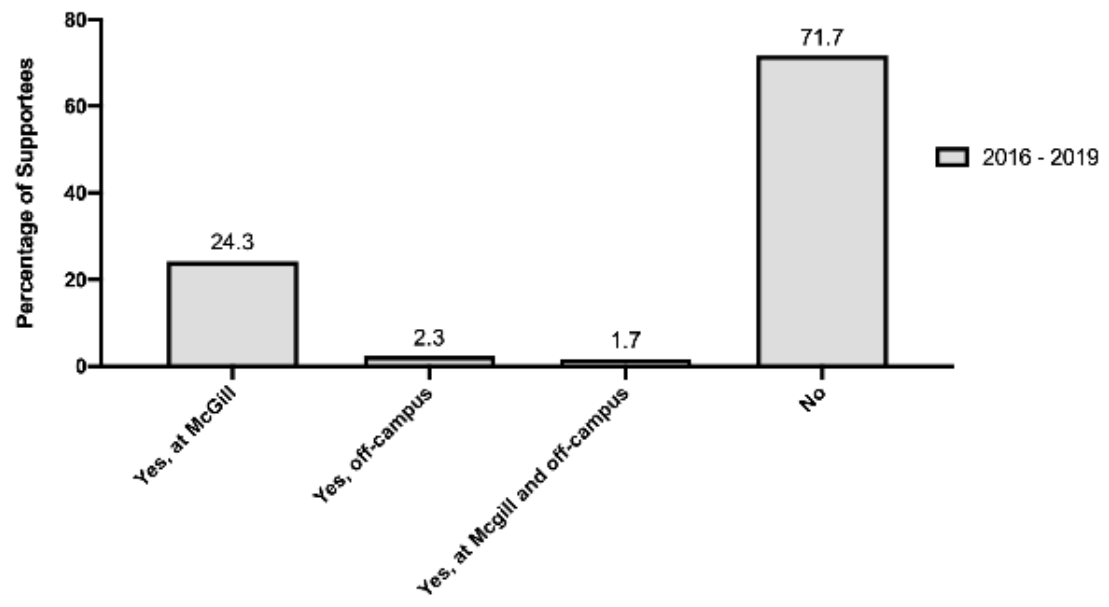

C.

Quality of PSC Compared to other Mental Health Services

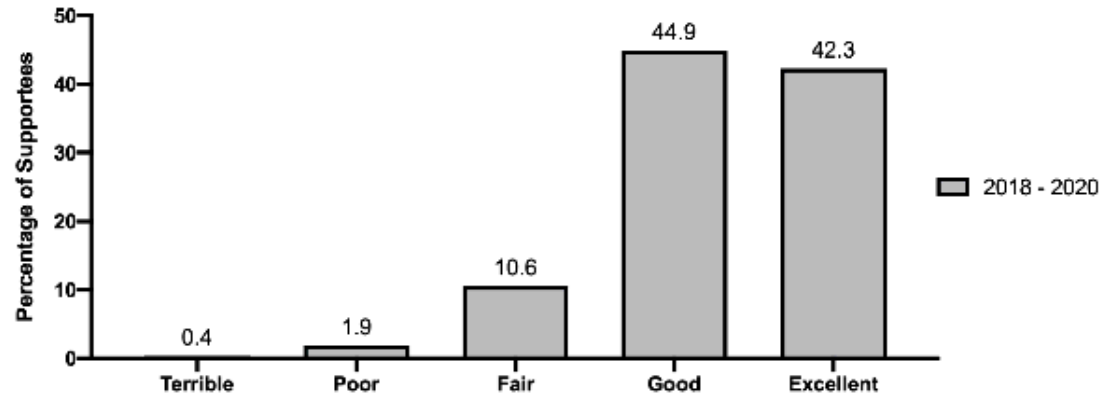

Figure 2

A) Percentage breakdown of supportees who use professional mental health services from 2016 - 2019.

B) Percentage breakdown of supportees who are on a waitlist at professional mental health services from 2016 - 2019. C) Percentage breakdown of how supportees would rate the quality of PSC compared to other mental health services. 


\section{Supplementary Files}

This is a list of supplementary files associated with this preprint. Click to download.

- SupplementaryData.docx 